\title{
Manipulations to reduce simulator-related transient adverse health effects during simulated driving
}

\author{
M. Jäger · N. Gruber · R. Müri · U. P. Mosimann • \\ T. Nef
}

Received: 13 March 2013 / Accepted: 19 May 2014 / Published online: 3 June 2014

(C) International Federation for Medical and Biological Engineering 2014

\begin{abstract}
User comfort during simulated driving is of key importance, since reduced comfort can confound the experiment and increase dropout rates. A common comfort-affecting factor is simulator-related transient adverse health effect (SHE). In this study, we propose and evaluate methods to adapt a virtual driving scene to reduce SHEs. In contrast to the manufacturer-provided high-sensory conflict scene (high-SCS), we developed a low-sensory conflict scene (low-SCS). Twenty young, healthy participants drove in both the high-SCS and the low-SCS scene for $10 \mathrm{~min}$ on two different days (same time of day, randomized order). Before and after driving, participants rated SHEs by completing the Simulator Sickness Questionnaire (SSQ). During driving, several physiological parameters were recorded. After driving in the high-SCS, the SSQ score increased in average by $129.4(122.9 \%, p=0.002)$ compared to an increase of $5.0(3.4 \%, p=0.878)$ after driving in the low-SCS. In the low-SCS, skin conductance decreased by $13.8 \%(p<0.01)$ and saccade amplitudes increased by $16.1 \%(p<0.01)$. Results show that the
\end{abstract}

M. Jäger · N. Gruber · R. Müri · U. P. Mosimann · T. Nef Gerontechnology and Rehabilitation Group, University of Bern, Murtenstrasse 50, 3010 Bern, Switzerland

R. Müri

Division of Cognitive and Restorative Neurology, Department of Neurology, Inselspital, University of Bern, Bern, Switzerland

U. P. Mosimann

Department of Old Age Psychiatry, University Hospital of Psychiatry, University of Bern, Bern, Switzerland

T. Nef $(\triangle)$

ARTORG Center for Biomedical Engineering Research, University of Bern, Bern, Switzerland

e-mail: tobias.nef@artorg.unibe.ch investigated methods reduce SHEs in a younger population, and the low-SCS is well accepted by the users. We expect that these measures will improve user comfort.

Keywords Driving simulator · Physiological measures · Eye-tracking

\section{Introduction}

One reason for the popularity of driving simulators (DS) is that laboratory-based DS are a safe way to test driving performance under standardized conditions [31]. Thereby, a person is immersed in a computer-generated virtual environment and interacts with the technical system [29]. The interaction happens via a sensor-controlled car model that provides input (i.e., steering wheel, pedals) and output modalities (i.e., vision, sound, vibration, motion).

Among many applications, DS are used to study driving behavior [32, 35], to train novice drivers [15], for fitness to drive assessments [6], and to test and validate new cockpit components [11].

User comfort during simulated driving is of key importance, since reduced comfort can alter the behavior of the user, confound data [33], limit the effectiveness of training [19], and increase dropout rates [9]. However, many users experience discomfort during and sometimes after a session in a DS $[8,9,20,25]$. There are many factors that influence comfort in a DS (for review see [44]). One of the most important is simulator-related transient adverse health effect (SHE), also known as simulator sickness or simulation adaptation syndrome. Kolasinski [30] maintain that SHE depends on individual user factors (i.e., age, gender, experience), task-specific factors (i.e., driving circuit, optical flow), and simulator-related factors (i.e., field 
of view, contrast). In the review of Brooks et al. [5], five theories about the pathophysiology of SHE are described. The most accepted theory that explains SHE is Reason and Brand's sensory conflict theory [41]: It proposes that SHE is the result of a mismatch between the sensory information of the visual and the vestibular system. Hence, the optical flow of the virtual scene indicates motion via the visual system, while the vestibular system does not perceive acceleration, which indicates no motion. Optical flow is the distribution of apparent velocities of movement of brightness patterns in an image [21]. In a fixed-based DS, optical flow is caused by relative motion of virtual objects on the screens and by user head movements [47]. As studied by Gibson [18], the amount of optic flow depends on the environment and might be an important contributor to SHE in fixed-based DS.

Symptoms of SHE can range from mild discomfort to severe and prolonged nausea, dizziness, and disorientation [22, 42]. Symptoms can appear within minutes and can cause discomfort for up to several hours [45]. Estimates of SHE incidence vary widely. Bertin et al. [3] reported $30 \%$, while in another study Kennedy [28] reported $88 \%$. Furthermore, it has been reported that $80 \%$ of all participants showed an increase of symptoms within 10 min after becoming immersed in a virtual environment and can arise from relative motion of objects and the viewer [9].

Several researchers [17, 26, 27] developed and adopted questionnaires as measures of SHE, and the Simulator Sickness Questionnaire (SSQ) became the most often used measure to quantify symptoms of SHE [43]. Others have combined the SSQ with a number of physiological measures and have found that simulator-induced symptoms are associated with increased heart [10] and respiratory rate [24], increased skin conductance [23], and decreased skin temperature [3]. Sweating is a common physiological manifestation of SHE and can be measured by an increased skin conductance. That is why besides SSQ, skin conductance is used in most studies on SHE as main outcome measure [2, $3,24]$. In addition, we measured fixation durations as well as saccade amplitudes using a head-mounted eye-tracking system. According to the sensory mismatch theory [5], SHE occurs when there is a mismatch between input to the visual system created by the optical flow and the vestibular system. Therefore, one might expect that participants would try to minimize retinal optical flow which could be achieved by less eye movements, more central fixations, and reduced saccade amplitudes. Hence, we expect to see a reduction in the saccade amplitudes as a compensatory mechanism to SHE. Therefore, it was hypothesized that fixation durations increase and saccade amplitudes decrease when SHE occur.

Prothero et al. [40] has put forth a new hypothesis which addresses how a stable background reference-an "independent visual background" (IVB)—would be used to enhance the observer's perception of the stable inertial frame of reference. The hypothesis was tested in a virtual environment using a head-mounted display to present a moving scene with an IVB and another scene without IVB to test SHE in healthy subjects [39]. The introduction of the IVB resulted in lower SSQ scores and improved user comfort. These effects were also confirmed in other studies (e.g., [12-14]). Lin et al. [34] added several IVB types to a driving simulator and evaluated the resulting impact in SHE. They found that after $2 \mathrm{~min}$ of driving exposure, subjects reported lower SSQ scores. Mourant et al. [37] investigated SHE and driving in four environments (country, suburban, city, and curves) using a fixed-base driving simulator. When driving on straight roads, subjects reported less SHE than driving in a city environment and on curves. They concluded that the rapid optic flow experienced in the driving simulator makes a substantial contribution to SHE. Yin and Mourant [47] studied the perception of optical flow when driving straight ahead and driving on curves. Results revealed that the amount of optical flow was highest when driving in curves and lowest when driving straight ahead. Thereby, the amount of optical flow was represented as the mean value of the sum over the number of image frames.

The purpose of this study was to investigate a combination of three methods to reduce the sensory mismatch during simulated driving and evaluate whether this reduces SHE in a fixed-based driving simulator.

\section{Methods}

\subsection{Participants}

Twenty (10 woman, 10 men) healthy participants were included in the study. None of the participants were taking any medication, nor suffered from any vestibular dysfunction. The mean age was 27.7 years $(\mathrm{SD}=2.9$, aged 24 32 years). All participants had comparable computer skills and were active drivers; none of them had experienced an immersion in simulated driving before. All participants wore their daily vision aids. The experiment was conducted in accordance with the latest Declaration of Helsinki and approved by the local ethics committee. Prior to inclusion, all participants gave informed consent.

\subsection{Procedure}

Starting point was a manufacturer-provided scene characterized by a high optical flow that is associated with a highsensory conflict (high-SCS). We applied three methods that lead to a new scene with reduced sensory conflict (lowSCS). The methods were (1) scene optimization aiming at 
a reduction in optical flow, (2) superimposing of an IVB to provide visual motion and orientation cues that match those from the vestibular receptors, and (3) decrease of brightness of lateral projection screen to further reduce optical flow. Both scenes and the three methods are described in the following section. In this study, we were investigating differences between the two different scenes (high-SCS and low-SCS), and therefore, the experiment took place on two different days but on the same time of day. Participants were advised to eat the same on both days. The order the participant was exposed to the high-SCS or low-SCS scene was randomized. Participants were given a verbal explanation and instructions on the electrode attachment, the eye-tracker, as well as the handling of the driving simulator. Prior to exposure to the virtual scene, participants completed a pre-immersion questionnaire and the SSQ [27]. To get baseline measures, participants were instructed to observe a frozen image of the virtual scene for $5 \mathrm{~min}$. Then, participants were exposed for $10 \mathrm{~min}$ either to the original or the adapted virtual driving scene. After the virtual drive, participants were advised to observe again a frozen image of the scene for $1 \mathrm{~min}$. Each participant filled out the SSQ again and completed a post-immersion questionnaire after detachment of sensors and eye-tracking system.

\subsection{Description and adaptations of the scenes}

The driving circuit is implemented in the virtual environment of the driving simulator. The virtual environment is composed of two elements:

- The scene that specifies the static characteristics of the simulation including the terrain, roadways, signage, buildings, and vegetation [16].

- The scenario that specifies the dynamic characteristics of a simulation. This includes what is to happen and where it is to happen. Thus, it binds together activities and places. A scenario is typically defined as a series of episodes with tightly controlled critical events interspersed with periods of free driving [16]. Thus, other traffic participants (i.e., cars, cyclists, pedestrians) are characterized by their position, velocity, behavior, and appearance.

Both scenes (low-SCS and high-SCS) incorporated a four-lane (two-lanes per direction) road with moderate traffic that included seven right and 11 left turns, three intersections, and three roundabouts. During the route, all participants encountered standardized driving conditions. Furthermore, participants had to react to standardized scenarios such as a deer jumping on the street from the right, an emergency braking of the car in front, a car abruptly leaving a parking place, and a child behind a pillar jumping in the street from the right border. The scenarios as well as the route of the low-SCS were exactly the same as in the high-SCS, but three methods were investigated to adapt the scene to reduce SHE:

1. First, the virtual scene was optimized aiming at a reduction in optical flow (Fig. 1). Optical flow depends on the virtual environment and objects such as building and trees close to the road generate more optical flow. Therefore, objects along roads (i.e., houses, street lamps) were removed and road side and surface were homogenized.

2. As a second modification, an IVB was implemented. The IVB is a component in the virtual scene that provides visual motion and orientation cues that match those from the vestibular receptors [14]. In our study, a static, thin ( $3 \mathrm{~mm}, 4$ cycles per radian), black grid IVB was superimposed over the entire virtual scene (Fig. 2).

3. Third, brightness of the lateral projection screens was decreased by $48 \%$ (Fig. 2) to further reduce optical flow. Thereby, the contrast of the lateral projection screens became decreased which leads to a decrease of optical flow [21].

\subsection{Apparatus}

\subsubsection{Driving simulator}

A fixed-based driving simulator (F12PI-3/A88, Foerst $\mathrm{GmbH}$ ) setup was used to create the simulation environment. Three projectors (Ultra Short Focus LCD projector, Sanyo) with $1,024 \times 768$ pixel resolution project the image onto three canvases $(1.80 \times 1.39 \mathrm{~m})$. The canvases form a $120^{\circ}$ angle and are positioned in front of the participant, creating a $180^{\circ}$ horizontal and $40^{\circ}$ vertical field of view. Three computers with Microsoft Windows 7 operating system (Microsoft Corp.) are used to control the simulation. One computer calculates and controls the dynamic scenario, and the other two computers render the graphics at $35 \mathrm{~Hz}$. To avoid interferences as well as distractions to the driver, the driving simulator is installed in a temperature-, light-, and noise-controlled room (Fig. 3).

\subsubsection{Sensory systems}

While the participant was immersed to the virtual world, eye movements, heart rate, respiratory rate, skin temperature, and skin conductance were measured. Eye movements were recorded by using a head-free eye-tracker with videobased/corneal reflection tracking (SMI iView X HED, $50 \mathrm{~Hz}$ ). Two cameras were used-one to capture the pupil and the corneal reflection and one to film the scene. The outcome variables of the eye-tracking measurements were 

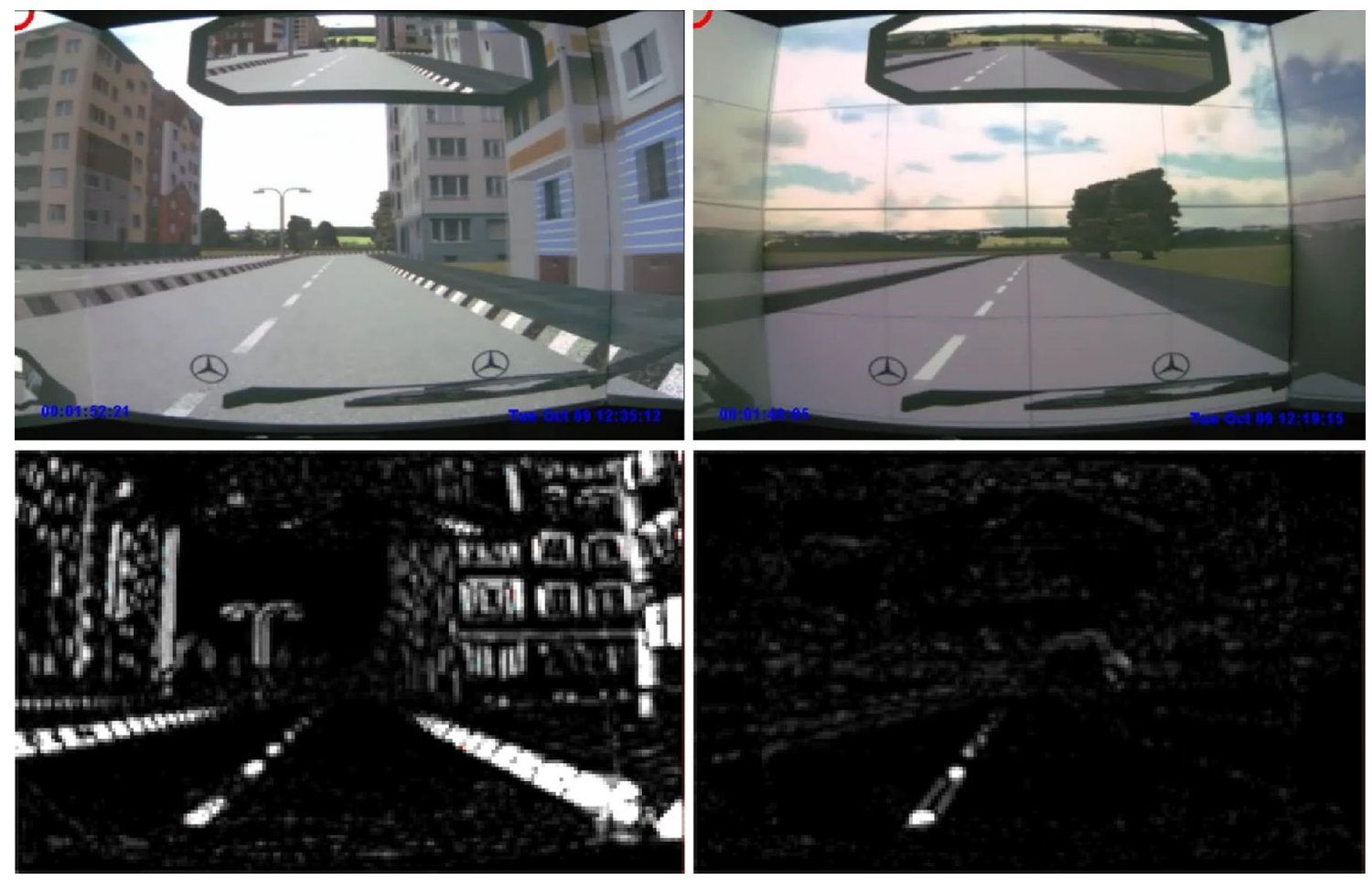

Fig. 1 Upper images show screenshots of the high-SCS (left) and low-SCS as participants are seeing them recoded with the scene

optical flow during $0.04 \mathrm{~s}$ ( 25 frames per second) of the two scenes is shown in gray scale. Optical flow was calculated using Horn and camera from the head-mounted eye-tracker. In the lower images, the

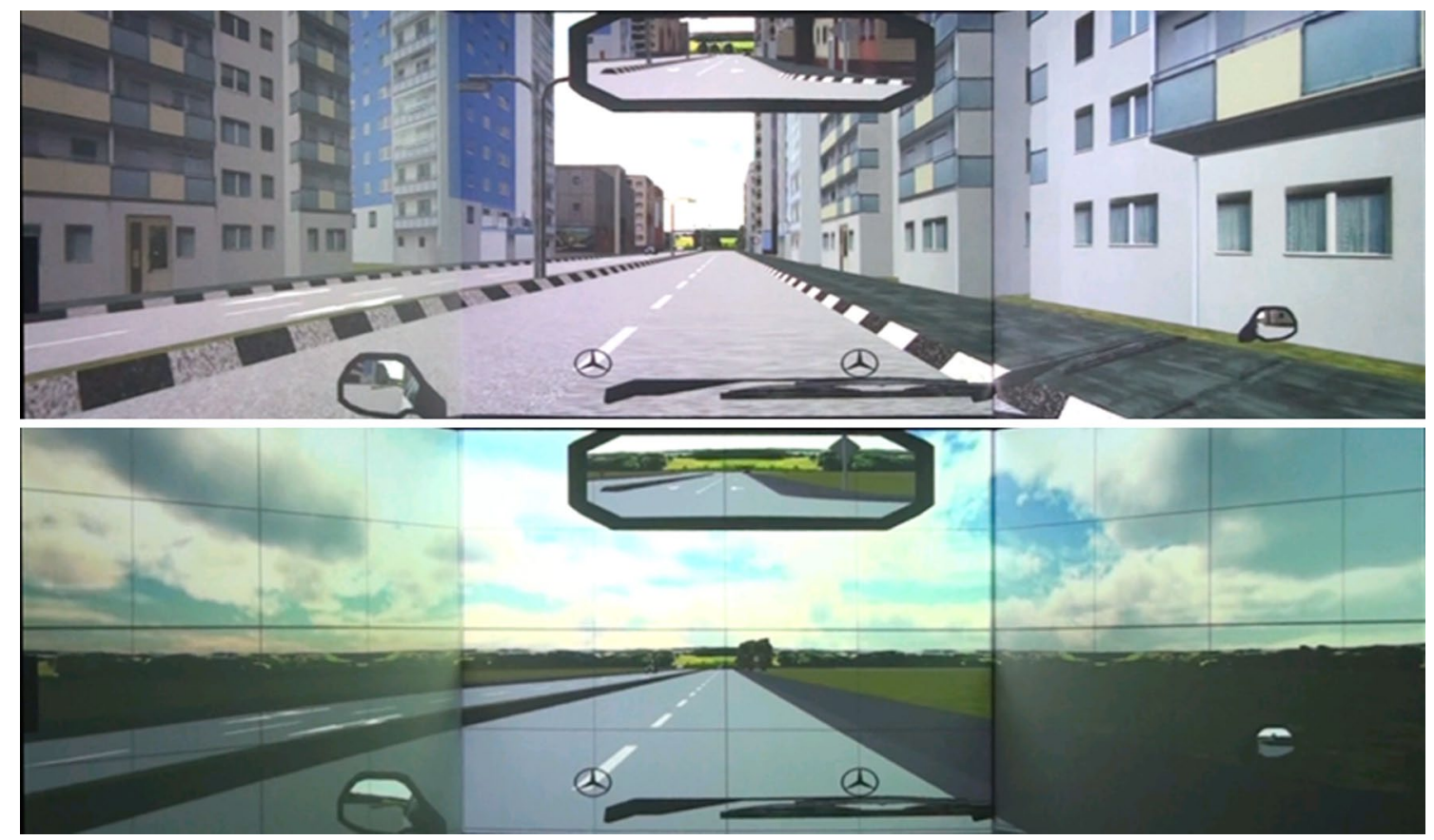

Fig. 2 Screenshots of high-SCS (upper image) and low-SCS scene. Low-SCS scene was optimized with respect to reduce optical flow and contains an independent visual background. Furthermore, brightness of the two lateral projection screens was decreased 


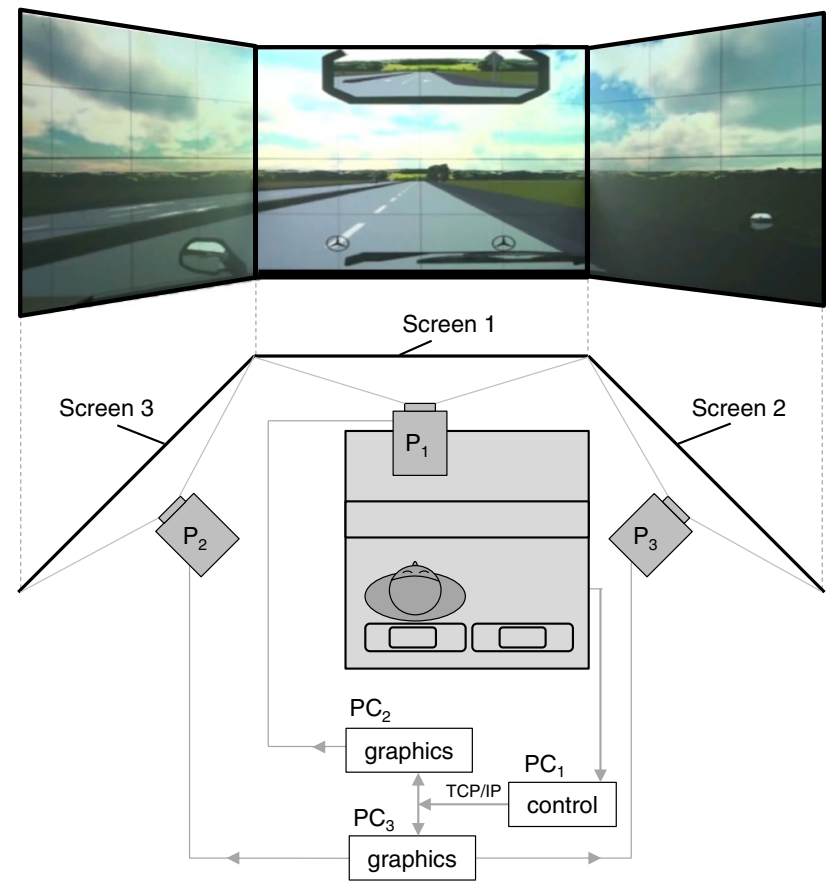

Fig. 3 Top-down view (schematic) of the experimental setup $\left(P_{1-3}\right.$ : projectors). $\mathrm{PC}_{1}$ controls the dynamic scenario, while $\mathrm{PC}_{2}$ and $\mathrm{PC}_{3}$ render the graphics at $35 \mathrm{~Hz}$. The participant was sitting in the driving simulator's chassis, which is based on authentic original design

fixation durations and saccade amplitudes, which were stored on a separate computer.

Heart rate was measured using a photoelectric pulse sensor for pulsatile blood flow (g.PULSEsensor, g.tec medical engineering $\mathrm{GmbH}$ ). The sensor was positioned at the volar surface of the distal phalanx of the index finger. Skin conductance was recorded with a galvanic skin response sensor (g.GSRsensor, g.tec medical engineering $\mathrm{GmbH}$ ) at the volar surfaces of the distal phalanxes of the third and fourth fingers of the left hand. A thermistor flow sensor (g.SLEEPsensor, g.tec medical engineering $\mathrm{GmbH}$ ) was used to get respiratory rate (nose and mouth). Skin temperature was measured using a thermistor YSI 400 (g.TEMPsensor, g.tec medical engineering $\mathrm{GmbH}$ ), which was attached at the volar surface of the distal phalanx of the fifth finger. Heart rate, respiratory rate, skin temperature as well as skin conductance were amplified with a DataLink amplifier (Biometrics Ltd.) and sampled with 1,000 Hz. Signals were not filtered before data analysis.

\subsection{Data analysis}

All data were calculated as mean values for time frames of $60 \mathrm{~s}$ for the experimental period of $10 \mathrm{~min}$ as well as $5 \mathrm{~min}$ before and 1 min after immersion. Matlab R2011a (The MathWorks, Inc.) was used to conduct the data analysis.
Mean duration of saccades and fixations as well as saccade amplitudes were calculated directly from the eyetracking system using the corresponding event marker (SMI). Minimal fixation duration was defined as $80 \mathrm{~ms}$. To quantify heart rate and respiratory rate, a low-pass filter was applied and a peak detection algorithm was used to count peaks per minute. Signal amplitudes were not taken into account. Skin conductance and temperature were continuous signals where mean values of every minute (amplitude) were calculated. Nonparametric Wilcoxon rank-sum test was used to test significance of the differences between the measures in the high-SCS and the low-SCS. Furthermore, linear regression was calculated to show dependency of the change in SSQ score and the change in saccade amplitudes.

\section{Results}

\subsection{Simulator Sickness Questionnaire}

All participants rated the SSQ before and after both the high-SCS and low-SCS scene. Figure 4 shows the mean values of the SSQ. Before driving in the high-SCS, mean SSQ score was 105.25 (SE 27.8), while the score was 148.01 (SE 26.8) before the low-SCS scene. After 10-min driving in the high-SCS, the SSQ score increased by $122.9 \%(p=0.002)$ compared to an increase of $3.4 \%$ ( $p=0.878$ ) after driving in the low-SCS. The difference of the change in SSQ between the high-SCS and the low-SCS was statistically significant with $p=0.005$.

Note that the results trend to differ among genders (Fig. 4, right). In the high-SCS, female subjects $(N=10)$ reported a mean increase of SHE by 160.98 points ( $p=0.038)$, while males $(N=10)$ rated SHE 97.81 points higher $(p=0.019)$. This difference showed a trend with $p=0.073$. In the low-SCS, female subjects rated SHE 24.98 points higher $(p=0.620)$, while in the male group, a decrease of 14.91 points $(p=0.748)$ was measured.

\subsection{Physiological measures}

In Table 1, mean values of different physiological measures are summarized for both scenes. While differences in heart rate, respiratory rate, and skin temperature were less noticeable, skin conductance was significantly lower and saccade amplitudes were higher in the low-SCS.

The mean saccade amplitudes during driving are in the low-SCS $16.1 \%(p<0.01)$ higher compared to the high-SCS (Fig. 5). Also, in the low-SCS scene, the mean fixation duration is $6.3 \%(p=0.081)$ smaller than in the high-SCS scene. In both scenes, the difference between before and during driving is not significant $\left(p_{\text {high }}=0.320\right.$, 


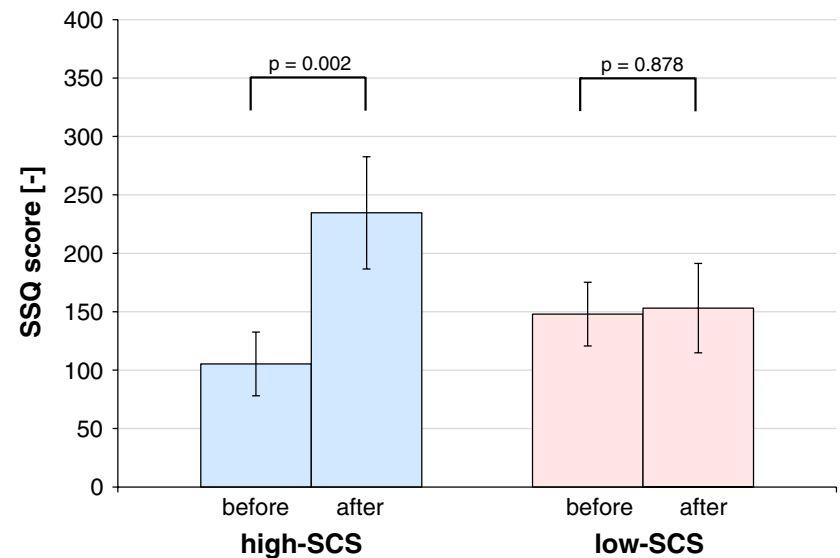

Fig. 4 Bar plots of mean values of the Simulator Sickness Questionnaire before and after both the high-SCS and low-SCS scene. Left plot represents scores of both genders $(N=20)$; right plot shows

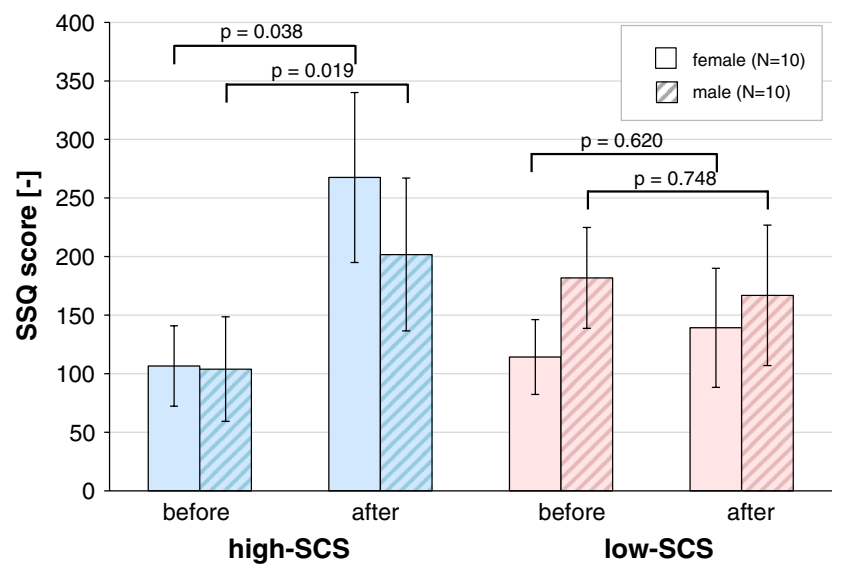

results female $(N=10)$ and male $(N=10)$ separately. Error bars represent the standard error

Table 1 Summary of the mean values and standard errors (SE) of the physiological measures recorded during simulated driving in the high-SCS and the low-SCS

\begin{tabular}{|c|c|c|c|c|c|c|c|}
\hline & \multicolumn{3}{|l|}{ High-SCS } & \multicolumn{3}{|l|}{ Low-SCS } & \multirow[t]{2}{*}{$p^{\mathrm{a}}$} \\
\hline & 5 min prior & During driving & 1 min after & 5 min prior & During driving & $1 \mathrm{~min}$ after & \\
\hline Fixation duration (ms) & $523.02(32.02)$ & $617.52(71.49)$ & $531.12(58.72)$ & $522.28(31.01)$ & $580.82(51.96)$ & $568.16(62.07)$ & 0.081 \\
\hline Saccade amplitude (deg) & $2.09(0.19)$ & $1.82(0.13)$ & $1.91(0.18)$ & $2.12(0.25)$ & $2.17(0.15)$ & $2.02(0.21)$ & $<0.01$ \\
\hline Heart rate (number/min) & $75.40(1.90)$ & $80.36(3.31)$ & $76.67(3.06)$ & $76.00(2.51)$ & $81.99(2.56)$ & $76.75(2.67)$ & 0.154 \\
\hline Respiratory rate (number/min) & $19.15(0.83)$ & $20.83(1.33)$ & $18.71(0.85)$ & $19.86(0.69)$ & $20.95(1.46)$ & $18.21(0.74)$ & 0.365 \\
\hline Skin conductance $(\mu \mathrm{S})$ & $3.29(0.03)$ & $3.40(0.04)$ & $3.40(0.05)$ & $3.28(0.03)$ & $3.36(0.03)$ & $3.36(0.04)$ & $<0.01$ \\
\hline Skin temperature $\left({ }^{\circ} \mathrm{C}\right)$ & $31.09(0.05)$ & $31.29(0.08)$ & $30.92(0.06)$ & $31.11(0.04)$ & $31.09(0.05)$ & $31.00(0.05)$ & 0.459 \\
\hline
\end{tabular}

Significant $\mathrm{p}$-values are in bold

${ }^{a} p$ value computed between high-SCS and low-SCS during driving $\left.p_{\text {low }}=0.671\right)$. Furthermore, the cumulated fixation time in the center of the image $\left(<10^{\circ}\right.$ eccentricity) versus the periphery $\left(>10^{\circ}\right.$ eccentricity) were calculated. In the lowSCS, the mean value and standard deviation of the time spent in the center are $58.3 \pm 6.6 \%$ versus the high-SCS $63.5 \pm 8.7 \%$ of the total cumulated fixation time. The difference of $5.2 \%$ is statistically significant with $p=0.006$.

Figure 6 shows the progress of skin conductance. At the beginning, skin conductance starts increasing significantly $(p<0.001)$ in both scenes and reaches a plateau. Compared to the high-SCS, in the low-SCS skin, conductance is $13.8 \%$ smaller $(p<0.01)$ during driving. No significant differences prior the experiment and during experiment were found for the other physiological measures.

\subsection{Correlation SSQ: physiological measures}

Linear regression was calculated between the change of SSQ score and saccade amplitudes as well as skin conductance. Strong correlation of $r=0.80$ was found for saccade amplitudes (Fig. 7) and weak correlation for skin conductance $(r=0.34)$. Positive SSQ scores ( $x$ axis) mean that symptoms of SHE increased during driving in the simulator, while scores $<0$ mean a decrease of symptoms. Since not the difference between the two scenes are of interest, the values of both the high-SCS and the low-SCS were taken into account $(N=40)$.

\section{Discussion}

The primary aim of this study was to describe and investigate a combination of three methods to evaluate whether they reduce SHE. The methods were (1) scene optimization aiming at a reduction in optical flow, (2) superimposing of an IVB, and (3) decrease of brightness of lateral projection screen to further reduce optical flow.

When comparing the SSQ score before and after driving in the simulator, in the high-SCS, the SSQ score increased by $122.9 \%$ while in the low-SCS, only a minor increase 


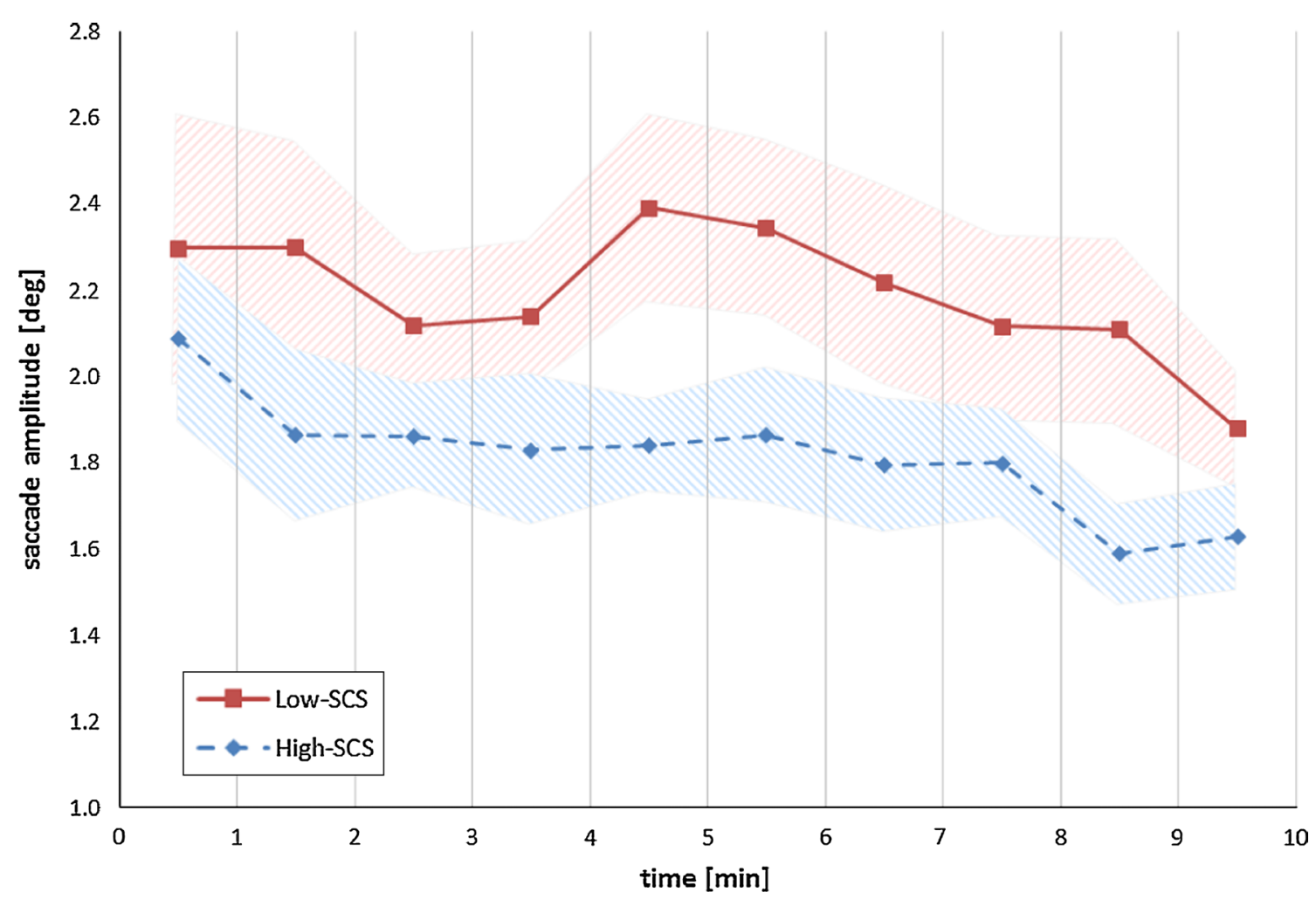

Fig. 5 Progress of saccade amplitudes during 10-min driving in the simulator. Mean values of each minute was calculated. Shaded area represents the standard error

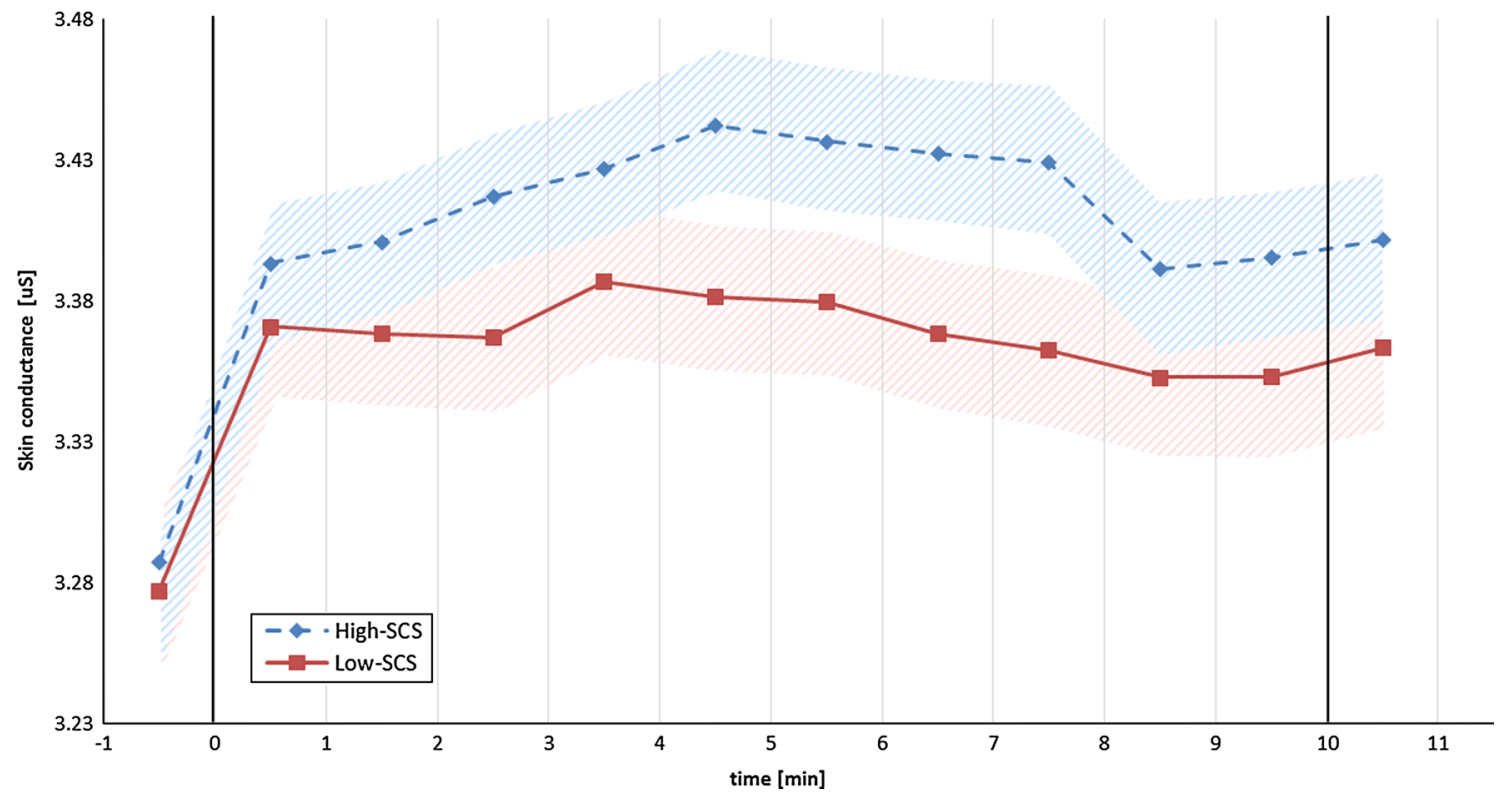

Fig. 6 Progress of skin conductance during 10-min driving in the simulator, and 1 min before and after immersion. Mean values of each minute was calculated. Shaded area represents the standard error

of $3.4 \%$ was observed. This result supports strongly the hypothesis that the combination of the three methods significantly reduces SHE. This is true for both genders, but the effects are more pronounced in female participants, who seems to be more susceptible to SHE and would benefit the most from the proposed methods to reduce SHE. 


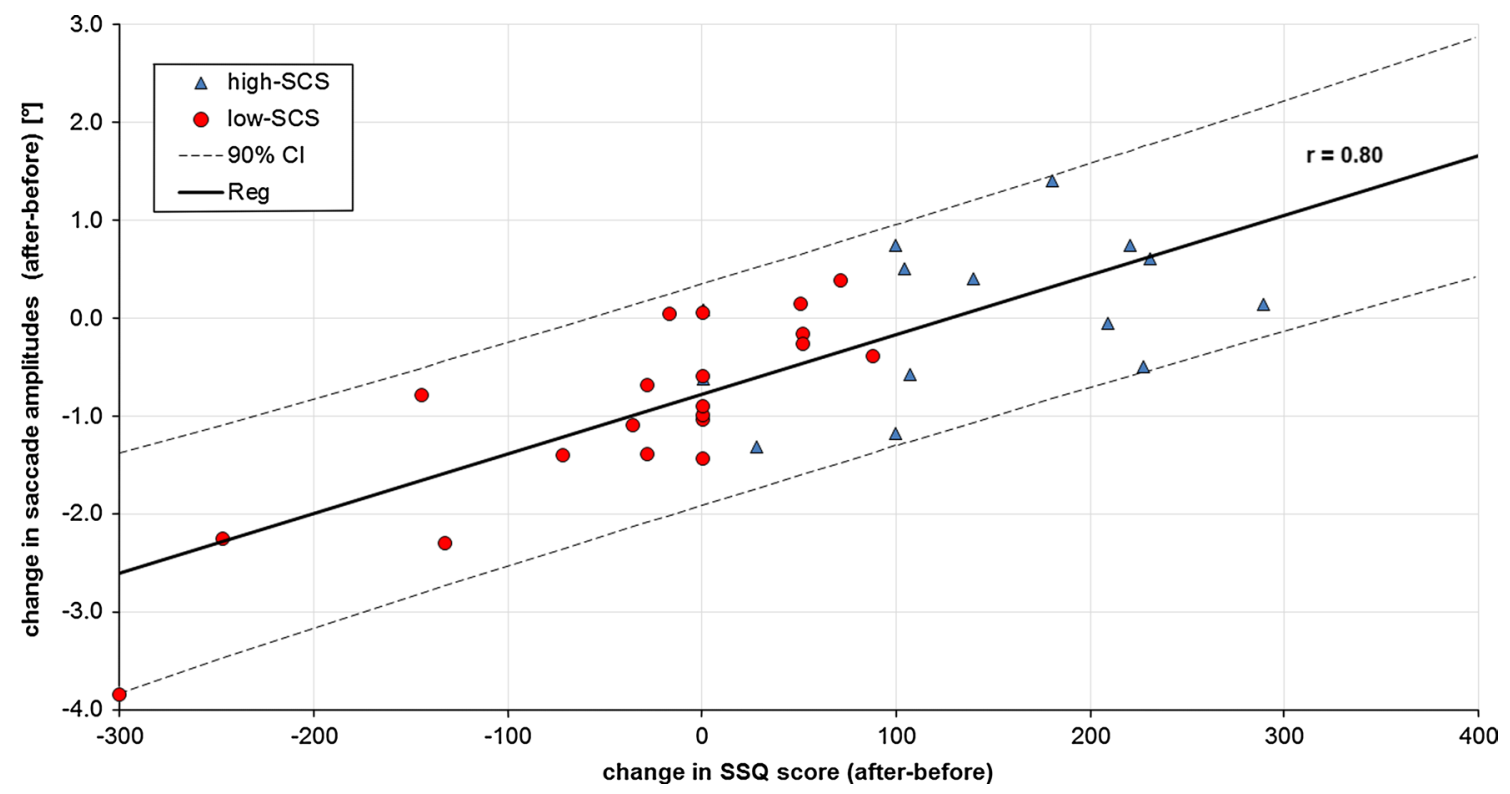

Fig. 7 Linear correlation between the change in SSQ score and the change in saccade amplitudes

The gender differences have also been noticed by others [1, $30,38]$. Note that the SSQ score before driving depends on participant's actual condition and was, therefore, not the same at the 2 days. In this study, the change in SSQ score caused by the immersion into a virtual driving scene was relevant.

Besides lower SSQ scores, all subjects also showed lower skin conduction when driving the low-SCS compared to driving the high-SCS (Fig. 7). The difference is significant and the correlation between SHE, SSQ, and skin conduction was also observed by others, who concluded that skin conduction is an important indicator for SHE [3, 29, 36]. The differences in heart rate, respiratory rate, and skin temperature between the two scenes are less noticeable than the difference in skin conductance. Also this has been observed by others, and Bertin et al. [3] concluded that skin conductance is probably the most reliable physiological SHE indicator.

In addition to these measures, we recorded fixation durations and saccade amplitudes. Saccade amplitudes showed significant differences between the high-SCS and the low-SCS, while fixation durations showed a trend. In the low-SCS, fixation duration is on average $6.3 \%$ shorter compared to the fixations duration when driving in the high-SCS. Furthermore, the saccade amplitudes are $16.1 \%$ higher in the low-SCS. Strong correlation between the change of saccade amplitudes and SSQ scores was found $(r=0.80)$. Compared to a correlation between skin conductance and SSQ, saccade amplitudes seem to be a reliable measure for SHE. No previous study has related saccade amplitudes to SSQ-assessed SHE. There are several explanations why SHE goes together with decreased saccade amplitudes. It could be that reducing eye movements is a compensation strategy of the visual system to reduce the amount of optical flow. Against this assumption speaks the saccadic omission that refers to a lack of awareness of the motion across the retina that is generated during a saccade $[7,46]$. This is the result of a visual saccadic suppression, which is the selective blocking of the visual processing during eye movements [46]. Another explanation for the reduced saccade amplitudes in the high-SCS is that under the influence of SHE, the subject tries to minimize the difference of the optical flow that is perceived via the left and the right hemifield of vision. This difference is smallest when looking at the center of the scene. That would also explain the reduced saccade amplitudes in the high-SCS. This assumption is in favor with our finding, that the cumulated fixation time spent in the center of the image $\left(<10^{\circ}\right.$ eccentricity) is $5.2 \%(p=0.006)$ smaller in the low-SCS compared to the high-SCS.

During the 10-min drive, the participants had to react to different scenarios: For example (1) react to a deer jumping on the street, including an emergency brake or (2) driving on a narrow road because of road works. While the former example (1) causes the skin conductance to increase, the second one (2) leads to a decrease of skin conductance and let the subject calm down. Between minute two and four of the simulated drive, there are more scenarios where the participant has to react to, which leads to an increase in skin conductance and a decrease in saccade amplitudes. This explains the drop of saccade amplitudes in the first minutes 
(see Fig. 5) in the low-SCS and the drop of skin conductance in high-SCS after $7 \mathrm{~min}$.

We asked the test subjects whether they were disturbed by the IVB, but none of them even noticed the IVB. We observed the same for the decreased luminosity of the two lateral projection screens. Also, we observed that the lowSCS was much better accepted by the test persons than the high-SCS.

The reduction of scene complexity might have an influence on the driving performance. Therefore, we compared in post-analysis the driving performance in both scenes, and total number of errors, speed variability, lateral acceleration, time on brake pedal, and time to collision were evaluated. None of these parameters differed significantly in both scenes.

The strength of this study is that the measurements were conducted on two different days, with at least 1 day in between, at the same time of day. Also, the participants were instructed to eat the same on the 2 days. Furthermore, the test population is homogenous in age, gender, computer skills, and driving experience. The combination of SSQ and physiological measures is an additional advantage.

A limitation of the study is that we applied a combination of three methods, and we cannot determine how much each individual method contributes to the observed reduction of SHE. In this paper, we combine three modifications to reduce SHE during simulated driving. While others have studied single modifications (i.e., IVB [39]), no study has measured the effects of a combination of the three modifications. In this work, we show that the combination of the three modifications has significant effects on SHE, and these modifications can be recommended to other researchers as well. For future studies, it would be interesting to study the effects of the individual modifications in single conditions. Prothero et al. [39] used an IVB only and reduced SSQ score by $19.8 \%$. When we calculate the delta of SSQ score in the low-SCS and high-SCS and compare them, we found a reduction of $96.1 \%$ which is statistically significant with $p=0.005$. With this in mind and based on our observations, we believe that the reduction of scene content has the greatest influence to reduce SHE. Furthermore, the decrease of brightness is in favor to avoid flicker perception, which has been linked to SHE [30]. Due to the fact that the peripheral visual system is more sensitive to flicker than the fovea [4], the decrease of brightness in the two lateral projection screens is beneficial to avoid SHE. However, for future research, the influence of each method should be analyzed. While the influence of the IVB was already shown by others $[12,14,34,39]$, the contribution of the decrease of brightness should be further investigated (particularly with regard to the influence on optical flow and flicker). In addition, the impact on training efficiency due to degraded virtual reality by superimposing an IVB and simplifying urban scenarios needs to be investigated. Furthermore, the medium-sized number $(N=20)$ could be a limiting factor of the validity of our study.

Susceptibility to SHE differs among age groups [30]. Therefore, for future work, it would be interesting to conduct a similar study in other age groups (i.e., with older participants). Moreover, the critical optical flow when subjects start to feel symptoms of SHE could be interesting and could contribute to optimizing our adaptations.

In summary, the results indicate that the combination of the three methods will reduce SHE in fixed-based DS, which may improve the user comfort and may reduce dropout rates. Therefore, we can recommend these methods to other researchers as well.

\section{References}

1. Allen RW, Park G, Cook M, Rosenthal TJ, Fiorentino D, Viirre E (2003) Novice driver training results and experience with a PC based simulator. In: Proceedings of the 2 nd international driving symposium on human factors in driver assessment, training and vehicle design

2. Bertin R, Collet C, Espié S, Graf W (2005) Objective measurement of simulator sickness and the role of visual-vestibular conflict situations. In: Driving simulation conference North America

3. Bertin R, Guillot A, Collet C, Vienne F, Espié S, Graf W (2004) Objective measurement of simulator sickness and the role of visual-vestibular conflict situations: a study with vestibular-loss (a-reflexive) subjects. In: Proceedings of Neuroscience meeting, San Diego

4. Boff KR, Lincoln JE (1988) Engineering data compendium: human perception and performance, vol 3. Armstrong Aerospace Medical Research Laboratory, Ohio

5. Brooks JO, Goodenough RR, Crisler MC, Klein ND, Alley RL, Koon BL, Logan WC Jr, Ogle JH, Tyrrell RA, Wills RF (2010) Simulator sickness during driving simulation studies. Accid Anal Prev 42(3):788-796

6. Brown LB, Ott BR (2004) Driving and dementia: a review of the literature. J Geriatr Psychiatry Neurol 17(4):232-240

7. Campbell FW, Wurtz RH (1978) Saccadic omission: why we do not see a grey-out during a saccadic eye movement. Vis Res 18(10):1297-1303

8. Classen S, Bewernitz M, Shechtman O (2011) Driving simulator sickness: an evidence-based review of the literature. Am J Occup Ther 65(2):179-188

9. Cobb SVG, Nichols S, Ramsey A, Wilson JR (1999) Virtual reality-induced symptoms and effects (VRISE). Presence Teleoper Virtual Environ 8(2):169-186

10. Cowings PS, Suter S, Toscano WB, Kamiya J, Naifeh K (1986) General autonomic components of motion sickness. Psychophysiology 23(5):542-551

11. Yong GI (2012) Effects of age and gender differences on automobile instrument cluster design. Advances in affective and pleasurable design. CRC Press, Boca Raton, FL, pp 22-212

12. Duh HB-L, Parker DE, Furness TA (2001) An independent visual background reduced balance disturbance envoked by visual scene motion: implication for alleviating simulator sickness. In: Proceedings of the SIGCHI conference on human factors in computing systems, Seattle, Washington, USA. ACM, pp 85-89 
13. Duh HBL, Abi-Rached H, Parker DE, Furness TA (2001) Effects on balance disturbance of manipulating depth of an independent visual background in a stereographic display. In: Proceedings of the human factors and ergonomics society annual meeting

14. Duh HBL, Parker DE, Furness TA (2004) An independent visual background reduced simulator sickness in a driving simulator. Presence Teleoper Virtual Environ 13(5):578-588

15. Fisher D, Pollatsek A, Pradhan A (2006) Can novice drivers be trained to scan for information that will reduce their likelihood of a crash? Inj Prev 12(Suppl 1):i25-i29

16. Fisher DL (2011) Handbook of driving simulation for engineering, medicine, and psychology. CRC Press, Boca Raton

17. Gianaros PJ, Muth ER, Mordkoff JT, Levine ME, Stern RM (2001) A questionnaire for the assessment of the multiple dimensions of motion sickness. Aviat Space Environ Med 72(2):115

18. Gibson JJ (1986) The ecological approach to visual perception. Routledge, London

19. Hettinger LJ, Berbaum KS, Kennedy RS, Dunlap WP, Nolan MD (1990) Vection and simulator sickness. Mil Psychol 2(3):171-181

20. Hill K, Howarth P (2000) Habituation to the side effects of immersion in a virtual environment. Displays 21(1):25-30

21. Horn BKP, Schunck BG (1981) Determining optical flow. Artif Intell 17(1):185-203

22. Howarth P, Costello P (1997) The occurrence of virtual simulation sickness symptoms when an HMD was used as a personal viewing system. Displays 18(2):107-116

23. Hu S, Grant WF, Stern RM, Koch KL (1991) Motion sickness severity and physiological correlates during repeated exposures to a rotating optokinetic drum. Aviat Space Environ Med 62(4):308-314

24. Johnson DM (2005) Introduction to and review of simulator sickness research. US Army Research Institute for the Behavior Sciences, Arlington

25. Kartiko I, Kavakli M, Ken C (2009) The impacts of animatedvirtual actors' visual complexity and simulator sickness in virtual reality applications. In: Sixth international conference on computer graphics, imaging and visualization

26. Kellogg RS, Kennedy RS, Graybiel A (1964) Motion sickness symptomatology of labyrinthine defective and normal subjects during zero gravity maneuvers. Aerosp Med 36:315-318

27. Kennedy RS, Lane NE, Berbaum KS, Lilienthal MG (1993) Simulator sickness questionnaire: an enhanced method for quantifying simulator sickness. Int J Aviat Psychol 3(3):203-220

28. Kennedy RS, Lane NE, Grizzard MC, Stanney KM, Kingdon K, Lanham S (2001) Use of a motion history questionnaire to predict simulator sickness. In: Driving simulation conference

29. Kim YY, Kim HJ, Kim EN, Ko HD, Kim HT (2005) Characteristic changes in the physiological components of cybersickness. Psychophysiology 42(5):616-625

30. Kolasinski EM (1995) Simulator sickness in virtual environments. Technical report of the U.S. Army Research Institute 1027

31. Lee HC, Cameron D, Lee AH (2003) Assessing the driving performance of older adult drivers: on-road versus simulated driving. Accid Anal Prev 35(5):797-803
32. Lee HC, Lee AH, Cameron D, Li-Tsang C (2003) Using a driving simulator to identify older drivers at inflated risk of motor vehicle crashes. J Saf Res 34(4):453-459

33. Lerman Y, Sadovsky G, Goldberg E, Kedem R, Peritz E, Pines A (1993) Correlates of military tank simulator sickness. Aviat Space Environ Med 64(7):619-622

34. Lin JJW, Abi-Rached H, Kim DH, Parker DE, Furness TA (2002) A "natural" independent visual background reduced simulator sickness. In: Proceedings of the human factors and ergonomics society annual meeting

35. Lukas A, Nikolaus T (2009) Driving ability and dementia. Zeitschrift fur Gerontologie und Geriatrie: Organ der Deutschen Gesellschaft fur Gerontologie und Geriatrie 42(3):205

36. Min BC, Chung SC, Min YK, Sakamoto K (2004) Psychophysiological evaluation of simulator sickness evoked by a graphic simulator. Appl Ergon 35(6):549-556

37. Mourant RR, Rengarajan P, Cox D, Lin Y, Jaeger BK (2007) The effect of driving environments on simulator sickness. In: Proceedings of the human factors and ergonomics society annual meeting

38. Mourant RR, Thattacherry TR (2000) Simulator sickness in a virtual environments driving simulator. In: Proceedings of the human factors and ergonomics society annual meeting

39. Prothero JD, Draper MH, Furness TA, Parker DE, Wells MJ (1999) The use of an independent visual background to reduce simulator side-effects. Aviat Space Environ Med 70(3):277-283

40. Prothero JD, Draper MH, Furness TA, Parker DE, Wells MJ (1997) Do visual background manipulations reduce simulator sickness. In: Proceedings of the international workshop on motion sickness: medical and human factors

41. Reason JT, Brand JJ (1975) Motion sickness. Academic Press, Oxford, $310 \mathrm{pp}$

42. Regan C (1995) An investigation into nausea and other sideeffects of head-coupled immersive virtual reality. Virtual Real 1(1):17-31

43. Schultheis MT, Rizzo AA (2001) The application of virtual reality technology in rehabilitation. Rehabil Psychol 46(3):296

44. Stanney KM, Mourant RR, Kennedy RS (1998) Human factors issues in virtual environments: a review of the literature. Presence 7(4):327-351

45. Ungs TJ (1989) Simulator induced syndrome: evidence for longterm after effects. Aviat Space Environ Med 60:252-255

46. Watson TL, Krekelberg B (2009) The relationship between saccadic suppression and perceptual stability. Curr Biol 19(12):1040-1043

47. Yin Z, Mourant RR (2009). The perception of optical flow in driving simulators. In: Proceedings of the 5th international driving symposium on human factors in driver assessment, training, and vehicle design 